\title{
Selbstverwaltung und Verfassungsrecht: Die italienische Erfahrung
}

\section{Tiefe Wurzeln: Der Einfluss verfassungsgerichtlicher Entscheidungen}

Zurückblickend auf die Ruinen des 2. Weltkriegs schien es der Verfassungsgebenden Versammlung klar, dass der Faschismus nicht nur eine Parenthese in Italiens Geschichte war. Die Schwäche liberaler Institutionen hatte die Ausbreitung eines totalitären Regimes ermöglicht. Die Justiz hatte wesentlichen Anteil an dieser Schwäche. Auswahl, Ernennung und Karriere der Richter waren abhängig von der Regierung; Vertreter der Exekutive ernannten die Gerichtspräsidenten - für alle Instanzen. Die Staatsanwälte waren dem Justizminister verantwortlich. Das Albertinische Statut (»Statuto Albertino« von 1848 - aus der Zeit des Königs Carlo Alberto) sah eine Reihe von formalen Garantien vor, aber es war leicht, diese zu umgehen. Hierfür waren mehrere Gründe verantwortlich; zu allererst ein nicht hinreichend striktes Verfassungsrecht und sodann die untrennbare Verflechtung von Justiz und Exekutive. Tatsächlich war Unabhängigkeit nicht mehr als ein Wort, nicht viel mehr als ein Traum. Staatsanwälte und Richter sahen tatenlos der schnellen und gewaltsamen Entwicklung des Faschismus zu.

Die Gründungsväter des neuen demokratischen Staates waren sich der Bedeutung der unauflösbaren, gegenseitig abhängigen Beziehung zwischen der Verwirklichung von Rechten und der Unabhängigkeit der Justiz bewusst. Der in der republikanischen Verfassung vorgesehene Consiglio Superiore della Magistratura (der Oberste Justizrat - nachfolgend CSM) ist etwas gänzlich anderes als das vor 1945 zuständige Organ gleichen Namens, sowohl hinsichtlich seiner Statuten als auch hinsichtlich seiner Zuständigkeiten.

Die Geschichte der Einführung und der Entwicklung des CSM ist eine außerordentlich wechselhafte Geschichte. Das Gesetz, das schließlich zur Schaffung des CSM führte, benötigte 10 Jahre (1958); die komplexen Beziehungen zwischen dem CSM und anderen Institutionen entwickelten sich im Verlauf von 30 Jahren, begleitet von Konflikten und Gerichtsentscheidungen. Das Verfassungsgericht spielte hierbei eine fundamentale Rolle: Am Anfang mit seinen Entscheidungen zur verfassungsrechtlichen Legitimation der für die Zuständigkeiten und Aktivitäten des CSM maßgeblichen gesetzlichen Regelungen; später durch seine Entscheidungen zu Zuständigkeitsstreitigkeiten zwischen den Institutionen (s. unten).

Verschiedene Entscheidungen des Verfassungsgerichts, einige von ihnen gehen zurück auf die ersten Jahre des CSM, verdeutlichen, dass die Bündelung aller Zuständigkeiten, die sich auf den Status von Richtern und Staatsanwälten beziehen, in Händen des CSM die Garantie der Unabhängigkeit und Autonomie der Justiz darstellt, jedoch keine vollständige Selbstverwaltung vorsieht (so kürzlich VerfassungsgerichtsE Nr. 379/92, unter Bezugnahme auf 168/1963, 44/1968, 142/1973 und 4/1986). Solch eine Zuständigkeit betrifft auch andere verfassungsrechtlich vorgesehene Organe, vor allem die Zuständigkeit des Justizministers (s. unten). Daher mag der Begriff »auto- 
nome Verwaltung « - da es nach den Ausführungen des Verfassungsgerichts unangemessen wäre, von »Selbstverwaltung « zu sprechen - eine bessere Vorstellung von der Komplexität der betroffenen institutionellen Beziehungen zu geben.

Unabhängig davon erklärte das Verfassungsgericht jedoch den ersten Absatz des Art. 11 des Gesetzes 195 vom 24.03.1958 für verfassungswidrig, insoweit dieser die Initiative für die Aktivitäten des CSM dem Justizminister zuerkannte und nicht dem CSM selbst. Diese Entscheidung war richtungsweisend für das Autonomiekonzept und die Rolle des CSM.

\section{Der Oberste Justizrat (CSM) und seine Struktur}

Heute besteht der CSM aus 24 gewählten Mitgliedern, von denen 16 von Richtern und Staatsanwälten selbst und 8 vom Parlament (in gemeinsamer Sitzung von Parlament und Senat) gewählt werden, plus 3 Mitgliedern kraft Amtes (Präsident der Republik, Präsident des Kassationsgerichts und Generalstaatsanwalt beim Kassationsgericht). Die parlamentarisch legitimierten Mitglieder (sogenannte Laienmitglieder im Gegensatz zu den Mitgliedern »in Robe«) werden unter Universitätsprofessoren (juristische Fakultät) und Rechtsanwälten mit jeweils längerer Berufserfahrung gewählt. Der Vizepräsident des CSM wird aus der Mitte der »Laienmitglieder « von allen Mitgliedern gewählt. In der Vergangenheit hatte das Parlament die Idee eines assoziativen Pluralismus favorisiert, durch den eine möglichst weit gefächerte Repräsentation dank eines proportionalen Wahlsystems mit verschiedenen Listen begünstigt wurde $(30 \mathrm{Ge}-$ wählte, davon 20 »n Robe« und 10 Laien plus 3 Mitglieder qua Amt). Dieses System hatte gleichzeitig bedeutet, dass die parlamentarische Opposition angemessen repräsentiert war. In den letzten Jahren wurde dieses System in ein weniger proportionales geändert, ohne Anwendung eines grundsätzlich konkurrierenden Wahlverfahrens, das verschiedene Listen mit unterschiedlichem politischen Hintergrund vorgesehen hätte.

Der CSM wird für vier Jahre gewählt, wobei die Gewählten nicht wiedergewählt werden können: Somit wird der CSM alle vier Jahre vollständig neu zusammengesetzt (mit Ausnahme der 3 Mitglieder kraft Amtes). Allerdings werden infolge der Gesetzesreform von 2002 (Gesetz 44 vom 28.03.02) die Mitglieder »in Robe« nicht mehr nach einem von Listen getragenen proportionalen Wahlverfahren gewählt, sondern durch Mehrheitswahl individueller Kandidaten auf regionaler Basis. Eine Reform, die den Einfluss von Richterorganisationen (die jeweils die Listen und entsprechende Programme präsentierten) zu reduzieren suchte, führte zu dem Ergebnis, dass jedes Bemühen um Meinungsbildung innerhalb einer assoziativen Organisationsform behindert wurde: Nur Kandidaten mit einer starken Unterstützung einer bestimmten Gruppe können nun noch hoffen, gewählt zu werden. Im Tandem mit dieser Entwicklung hat die Reduzierung der Anzahl der Laienmitglieder (folglich auch deren Repräsentanz) zusammen mit dem Wachsen einer tendenziellen politischen Mehrheit Ergebnisse produziert, die das Gegenteil dessen bedeuten, was als Reformabsicht angepriesen worden war. Die parlamentarisch gewählten Mitglieder agieren so letztlich nur noch als Repräsentanten einer politischen Mehrheit und nicht mehr als Persönlichkeiten von hohem wissenschaftlichen oder beruflichen Ansehen. 
Eine solche Erfahrung mag als Warnung vor möglichen desaströsen Ergebnissen dienen, wenn einmal die Logik der Mehrheit in ein autonomes Gremium Einzug gehalten hat, und außerdem die Folgen einer Entscheidung zugunsten eines bestimmten Wahlsystems vor Augen führen.

\section{Gründe für eine einheitliche Behandlung von Richtern und Staatsanwälten}

Der CSM ist ein gemeinsames Gremium für Richter und Staatsanwälte. Der Grund, weshalb die Verfassung ein einziges (für Richter und Staatsanwälte gemeinsames), autonom agierendes Organ vorsieht, ist in der dem Staatsanwalt zugewiesenen Rolle zu sehen, dank der er rechtsstaatliche Autorität im striktesten Sinne besitzt (also mit rechtspolitischer Macht ausgestattet ist, die auf verfassungsrechtlich geschützten Interessen, wie z. B. »persönlicher Freiheit« beruht). Dies bedeutet eine eindeutige Verknüpfung mit dem Verfassungsprinzip der Strafverfolgung als Auftrag. Sanktioniert durch Art. 112 der Verfassung bedeutet dieses Prinzip keineswegs die Verpflichtung, in allen Fällen von Rechtsverletzungen einzuschreiten. Tatsächlich stellt dieses Prinzip ein Kriterium dar, Verantwortung für die Optionen von Strafverfolgungsmaßnahmen zuzuweisen: Strafverfolgungsmaßnahmen verbleiben in einem solchen System vollständig innerhalb der Rechtsprechungsaufgabe und sind gemäß der durch Gesetz vorgegebenen Kriterien auszuüben. Aus diesem System hat das Verfassungsgericht eine Reihe von Verknüpfungen abgeleitet (einige davon betreffen das Gesetzessystem als Ganzes), die die Rolle des Staatsanwalts als die eines unabhängigen und autonomen Organs hervorheben.

Diese Prämissen sind entscheidend für ein grundlegendes Verständnis des Prozesses der Verfassungsrevision, wie sie heute diskutiert wird: Eine Revision, die (und dies ist keineswegs zufällig) einen Zusammenhang der Reform des CSM mit der Erosion des Strafverfolgungsprinzips und der angestrebten klaren Trennung von Richtern und Staatsanwälten verdeutlicht. Tatsächlich war diese Entwicklung schon seit 1999 auf dem Wege, und zwar mit der Neuformulierung des Verfassungsartikels 111, mit der die Natur des Richters als die eines »dritten Spielers« betont wird, deren Wesensmerkmal es sei, sowohl die gleiche als auch eine davon unterschiedliche Ausgestaltung der Unabhängigkeit im Vergleich zum Staatsanwalt vorzusehen.

\section{Die Kompetenzen des Obersten Justizrats (CSM)}

Seine heterogene Zusammensetzung, unter anderem dank der durch das Parlament gewählten Mitglieder, und die Tatsache, dass es sich um ein gewähltes Organ handelt, dessen Vorsitz der Präsident der Republik innehat (der von der italienischen Verfassung als ihr oberster Garant angesehen wird), sind ausnahmslos Faktoren, die zusammengenommen den Anspruch rechtfertigen, der CSM trage die Verantwortung für die Justiz nicht allein im Sinne einer bürokratisch umrissenen Managementaufgabe für bestimmte Bereiche, sondern auch als Hauptgesprächspartner in Verhandlungen mit anderen politischen Institutionen. Dieser Charakter des CSM wird durch seine Zusammensetzung und die ihm zugewiesenen Zuständigkeiten bestimmt. 
Die verschiedenartige Zusammensetzung des CSM (von den Kollegen gewählte Richter und Staatsanwälte, vom Parlament gewählte Berufsjuristen sowie der Präsident der Republik als Vorsitzender) und seine Zuständigkeiten hängen außerdem zusammen mit der Art und Weise, wie die Verfassungsgebende Versammlung die demokratische Legitimation dieses Organs, und allgemeiner der Justiz schlechthin, gesehen hat. Die Entscheidung der Verfassungsgebenden Versammlung zielte auf die Beantwortung der fundamentalen Frage: quis custodiet custodes? Demokratie wird nicht nur charakterisiert durch die Maßgeblichkeit des Willens der Mehrheit und den Vorrang des Gesetzes: Demokratie bedeutet auch - und dies in einem fundamentalen Sinne Verfahrensregeln vorzusehen, die den Willensbildungsprozess der Bürger kanalisieren, Minderheitenschutz garantieren und die Grundrechte des Verfassungsvertrags schützen.

Tatsächlich hat das Verfassungsgericht erklärt, dass die Definition, der CSM »repräsentiere in einem technischen Sinne die Justiz«, dahingehend ausgeführt werden müsse, dass die Anwesenheit von Laien im CSM in Verbindung mit der Verfassungsvorgabe hinsichtlich des Vorsitzes »die Antwort gebe auf die (von den CSM-Mitgliedern selber so gesehene) Notwendigkeit, eine sich selbst als separate Organisationseinheit darstellende Justiz zu vermeiden.« Daher also »seien angemessene Verfahren und Maßnahmen für die ständigen Beziehungen mit dem einheitlichen Staatsgefüge vorzusehen und aufrecht zu erhalten, ohne jedoch die erläuterte und garantierte Autonomie und Unabhängigkeit des CSM zu gefährden« (VerfassungsgerichtsE Nr. 142/ 1973).

Mit grundlegendem Beschluss vom 28.10.1982 reformierte der CSM seine internen Verfahrensregeln dahingehend, dass die Öffentlichkeit zu den Sitzungen des CSM zugelassen wurde (nur Fälle ausgenommen, in denen die Notwendigkeit des Schutzes der Privatsphäre eines Richters bzw. Staatsanwalts oder dritter Parteien vorrangig sind), und verdeutlichte damit die dem CSM durch die oben zitierte Verfassungsgerichtsentscheidung zugesprochene Rolle.

Art. 105 der Verfassung weist dem CSM die Zuständigkeit für alle die Richter bzw. Staatsanwaltschaft betreffenden »Ernennungen, Funktionszuweisungen, Versetzungen, Beförderungen und Disziplinarmaßnahmen« zu.

Die in der Verfassung genannten Zuständigkeiten beschreiben nicht abschließend die Rolle, die dem CSM von der Verfassung als einem mit verfassungsrechtlichen Garantien ausgestatteten Organ zugewiesen wird, um die Autonomie der Justiz als einer von allen übrigen staatlichen Gewalten unabhängigen Ordnungsmacht zu sichern (VerfassungsgerichtsE Nr. 44/1968) und außerdem vor Eingriffen anderer staatlicher Gewalten zu schützen, damit die Rechtsprechungsaufgabe unabhängig ausgeführt werden kann. Es hat immer wieder Versuche gegeben, die Rolle des CSM einzuschränken. Die für die korrekte Anwendung der Verfassung vorgegebenen Verfassungsregeln, Verfahren und Institutionen (wie z. B. das Verfassungsgericht) haben sich als unverzichtbar für die Bewahrung des Systems einer autonomen Organisation der Justiz erwiesen. 


\section{Die Ursachen der fortwährenden Konflikte mit anderen Gewalten}

Es ist schwierig, den institutionellen Streit in allen Nuancen zu erfassen, ohne ihn im größeren Zusammenhang mit der Frage nach einer »actio finium regundorum « zu betrachten; diese Frage nach den Kompetenzbereichen der einzelnen Staatsgewalten ist in den letzten 20 bis 30 Jahren zunehmend diskutiert worden. Ab 1980 hat die Justiz eine profilierte Rolle insbesondere in den äußerst umfangreichen Ermittlungen infolge von Mafia- und Terroraktionen gespielt (mit allen offensichtlich politischen und institutionellen Verwicklungen), und später, in den frühen neunziger Jahren, in den Ermittlungen, die die Organisation des politischen Systems in Italien und deren politische Führung unmittelbar betrafen (Strafverfolgungsmaßnahmen unter dem Stichwort $»$ mani pulite «).

Im Umgang mit dem organisierten Verbrechen und der Korruption hat Italien außerordentliche Erfolge erzielt; in den achtziger und neunziger Jahren sind zum ersten Mal in der Geschichte Mafia-Organisationen wie z.B. die Cosa Nostra ernsthaft getroffen worden, mit Tausenden von verurteilten Straftätern, die ihre Strafen auch tatsächlich abbüßten, mit der Ergreifung Hunderter Flüchtiger (einige von ihnen seit Jahrzehnten), durch die Konfiszierung von Geldbeträgen in Billionenhöhe, die nun zum Nutzen der Bürger eingesetzt werden konnten. Ein hoher Preis an Menschenleben wurde dafür gezahlt. Im Zusammenhang mit dem ersten Cosa Nostra-Prozess wurden der Leiter der Staatsanwaltschaft von Palermo, Costa, der Leiter der Untersuchungsrichter, Chinnici, mit zwei Polizisten seiner Eskorte, der Untersuchungsrichter Falcone mit seiner Ehefrau und vier eskortierenden Polizisten, der Untersuchungsrichter Borsellino mit fünf begleitenden Polizisten, der Generalstaatsanwalt Scopellini im Kassationsgericht und einige Regierungsbeamte wie Montana und Cassarà ermordet.

Die genannten Erfolge wurden ohne Beeinträchtigung verfassungsmäßiger Freiheiten und Garantien erzielt, sogar in jenen Tagen, als es nahezu unmöglich war, das Fernsehen einzuschalten, ohne Berichte über Blutbäder und Bombenanschläge in unseren Städten zu sehen, oder zu Zeiten, als die Zahl der durch das organisierte Verbrechen in Italien begangenen Morde nur von der Anzahl der Morde in Kolumbien überboten wurde.

Es ist schwierig, sich vorzustellen, dass solch eine Reihe von Erfolgen in derartig sensiblen Bereichen ohne das Primat der Unabhängigkeit (gerade auch für Staatsanwälte), die Befreiung der Richter und Staatsanwälte von interner Hierarchie sowie das vom CSM gebotene Schutzschild gegenüber einer politischen Macht, die beinahe täglich aggressiver gegenüber der Justiz agierte, hätte erreicht werden können.

Diese komplexen Fragen können hier nicht in der gebotenen Tiefe erörtert werden, aber es sollte kein Zweifel daran bestehen, dass diese Zusammenhänge zugleich Prämisse und Hintergrund für jede aktuelle Diskussion über die Zuständigkeiten des CSM und seiner durch die Verfassung vorgegebenen Rolle darstellen.

Als der CSM in früheren Jahren Maßnahmen ergriff, die geheime Loge der Freimaurer an einer Durchdringung und Beeinflussung der Justiz zu hindern, hatten die Spannungen einen ihrer Höhepunkte erreicht. Vor Verabschiedung des Gesetzes aus dem Jahre 1982, das sich mit der Mitgliedschaft in geheimen Organisationen befasste, 
war der CSM die einzige staatliche Autorität, die entschieden auf die Entdeckung der Existenz der geheimen Organisation mit dem Namen »Propaganda Loge 2 «(P2) reagierte. Gegen Richter und Staatsanwälte, die Mitglieder der P2-Loge waren, wurden Disziplinarverfahren durchgeführt, die mit härtester Bestrafung oder mit der persönlichen Entscheidung der Betroffenen, den Dienst selbst zu quittieren, endeten.

In einem anderen richtungsweisenden Fall, ausgelöst durch die öffentlichen Äußerungen des früheren Premierministers Bettino Craxi über Ermittlungsverfahren in Korruptionsfällen, brach der Konflikt erneut aus; wiederum wurde die Legitimation des CSM, sich mit der Sache zu befassen, in Frage gestellt. Konfrontiert mit der Entschlossenheit des CSM, sich dennoch der Angelegenheit anzunehmen, befahl der Präsident der Republik, den Sitzungssaal, in dem der CSM tagte, durch bewaffnete Kräfte (Carabinieri) zu besetzen; jedoch wurde der Befehl infolge starken Protestes der Politik und Öffentlichkeit aufgehoben, sodass der CSM seine Debatte schließlich fortsetzen konnte.

Die daraus resultierende Entschließung des CSM bestätigte seine verfassungsmäßige Aufgabe als Garant für die Unabhängigkeit der Justiz als Ganzes und die individuelle Unabhängigkeit des Richters bzw. Staatsanwalts (eben auch von äußeren Einflüssen »aus welcher Richtung auch immer«, Entschließung vom 19.12.1985).

Diese Prinzipien wurden noch einmal in einer Phase bestätigt, in der sich neuerlich ein feindseliges Klima gegenüber der Unabhängigkeit der Rechtsprechung entwickelte. Der Vorwurf gegenüber dem CSM lautete, den in den letzten Wahlen zum Ausdruck gekommenen Willen des Volkes nicht hinreichend berücksichtigt zu haben, weil der CSM mit seiner Entschließung vom 18. Juni 2003 argumentiert habe, »wenn das Wesen und der Wert einer konstitutionellen Demokratie auf der Souveränität des Volkes beruhen, ... ergibt sich zusätzlich aus dem Gesetzesvorbehalt, dass die institutionellen Garanten der Gewaltenteilung (hier die rechtsprechende Gewalt) ... ihre Legitimität unmittelbar aus der Verfassung herleiten«. Dies bedeutet für die Rechtsprechung, dass sie in erster Linie den in den Gesetzen zum Ausdruck kommenden Willen des Volkes und nicht den Willen des Volkes unmittelbar, wie auch immer er z. B. in Wahlen zum Ausdruck kommen mag, zu befolgen hat. Sie hat immer auch dann die durch Verfassung und Gesetze garantierten schutzwürdigen Interessen von Minderheiten zu beachten, wenn die weit überwiegende Mehrheit der Bevölkerung hinsichtlich bestimmter Minderheiten andere Meinungen äußert oder durch Wahlergebnisse manifestiert haben mag.

\section{Das Verhältnis des Obersten Justizrats (CSM) zur Legislative}

Das Verhältnis zur Legislative ist ein interessanter Komplex bei der Betrachtung der verfassungsmäßigen Rolle des CSM und seiner Legitimität in der demokratischen, durch die Verfassung vorgesehenen Gewaltenteilung. Die derzeit diskutierten Probleme betreffen die Grenzen der Interaktionen zwischen den beiden Gewalten.

Die erste streitige Frage ergibt sich aus der Interpretation des Artikels 10 des Gesetzes Nr. 195/1958. Dieser formuliert ausdrücklich, dass der Justizminister die Meinung des CSM zu Gesetzesinitiativen der Regierung, die Angelegenheiten der Justiz betref- 
fen, einholen soll. In diesem Zusammenhang wird die Frage diskutiert, ob der CSM in seiner Stellungnahme auch auf im Parlament diskutierte Änderungen des eingebrachten Gesetzesentwurfs eingehen darf.

Die zweite Frage betrifft die Beziehungen zwischen den einzelnen Staatsgewalten, insbesondere jene zwischen dem CSM und dem Parlament, sowie die verfassungsmäßige Zuständigkeit des Justizministers für die Organisation der Justiz (siehe unten). Der Justizminister zeichnet die Erlasse des Präsidenten der Republik gegen, welche die Beschlüsse des CSM umsetzen. Dieses strukturelle Verhältnis der drei Organe (Präsident der Republik, in seiner Eigenschaft als Staatsoberhaupt - nicht als Vorsitzender des CSM -, CSM, Justizminister) leitet sich aus der Tatsache ab, dass die beiden zuerst genannten Organe nicht politisch verantwortlich sind, und führt so zu einer Reihe von institutionellen und interpretatorischen Problemen, die hier nur summarisch betrachtet werden können.

Es mag der Hinweis genügen, dass Möglichkeiten eines direkten Dialogs zwischen dem CSM und dem Parlament entwickelt wurden; die Legitimation des CSM als direkter Gesprächspartner des Parlaments in wichtigen Justizangelegenheiten wurde anerkannt; dennoch wurden die Stellungnahmen des CSM auch in den Fällen jeweils dem Justizminister mitgeteilt, wenn es sich um vom Parlament initiierte Gesetzgebungsvorhaben handelte. Dieser Problembereich wurde im Jahr 1968 durch einen Kompromissbeschluss geregelt, der für einige Jahre gut funktionierte. Erst zu Zeiten der zweiten Regierung Berlusconi wurde dieser Kompromiss durch den Justizminister Castelli in Frage gestellt. Im Jahre 1968 hatte ein Senatsbeschluss in Unterstützung einer Entscheidung des Staatspräsidenten Saragat festgelegt, dass der CSM bei einer derartigen Konstellation politisch exponiert ist (und folglich auch verantwortlich ist) und daher seine Stellungnahmen über Justizangelegenheiten an beide Häuser (Parlament und Senat) zu richten hat, aber jeweils über den Justizminister. Seitdem ist dies auch immer so gehandhabt worden, und die Stellungnahmen des CSM wurden immer mit beträchtlichem Interesse geprüft, wenn Regierungs- oder Parlamentsentwürfe zur Debatte standen.

Dennoch weigerte sich der Justizminister Roberto Castelli, die Stellungnahme des CSM aus 2003 an das Parlament weiterzuleiten. Der Sprecher des Senats legte offiziell Beschwerde beim Staatspräsidenten ein, weil der CSM seine Stellungnahme an die Sprecher des Parlaments und des Senats habe übermitteln lassen.

Ganz aktuell ist eine ähnliche Konstellation unter einem neuen Gesichtspunkt aufgetreten. Der CSM behandelte in einer seiner Stellungnahme sowohl die von der Regierung vorgeschlagenen Gesetze als auch die im Parlament dazu diskutierten Änderungsvorschläge. In diesem Zusammenhang hat der CSM außerdem Zweifel an der Legitimität eines in Frage stehenden Gesetzes geäußert. So etwas ist in zahlreichen Fällen in der Vergangenheit geschehen, und wiederholt ist dabei die Frage diskutiert worden, ob und wie eine Stellungnahme zu formulieren sei, wenn sie die Meinung äußert, der vorliegende Gesetzestext entspreche nicht der Verfassung. Ein starke parlamentarische Mehrheit protestierte vehement gegen derartige in Stellungnahmen des CSM geäußerte Auffassungen und ließ durch ihre führenden Vertreter - sogar unterstützt durch einzelne Juristen - verkünden, allein das Verfassungsgericht sei autori- 
siert zu beurteilen, ob ein Gesetz verfassungsgemäß ist: Als wäre das Äußern einer Meinung gleichbedeutend mit einer verbindlichen Feststellung über die Verfassungsmäßigkeit eines Gesetzes. Unter anderem haben auch diese öffentlichen Diskussionen die Regierungsmehrheit veranlasst, den CSM in der oben erläuterten Form zu reformieren.

\section{Der Oberste Justizrat (CSM) als eine sich selbstverwaltende Körperschaft - Das Verhältnis zwischen dem Präsidenten der Republik, der Versammlung des CSM und dem Vizepräsidenten}

Die oben beschriebene Debatte von 1985 war Anlass für die Erörterung des Verhältnisses zum Präsidenten der Republik (in seiner doppelten Eigenschaft als Präsident des CSM und als Staatspräsident) bei der Festlegung der Geschäftsordnung - eine scheinbar unbedeutendere Angelegenheit, die indessen Grundfragen der Rolle des CSM sowie dessen notwendiges Selbstverständnis im Hinblick auf effektive Interessenwahrnehmung im Einklang mit den ihm durch die Verfassung zugewiesenen Aufgaben berührt.

Das Recht, seine Angelegenheiten in eigener Zuständigkeit zu regeln, ist in der Rolle des CSM angelegt. Er ist daher ermächtigt, sich selbst eine Geschäftsordnung zu geben, wenn auch mit Zustimmung des Präsidenten. Das Recht, im Streifalle über die Rechtmäßigkeit der eigenen Maßnahmen selber zu entscheiden, ist indessen nicht in dem Recht auf Selbstverwaltung enthalten. Das Verfassungsgericht hat betont, dass die Rolle des CSM als Garant (»ein Organ mit signifikanter verfassungsrechtlicher Bedeutung «) seine Maßnahmen nicht vom allgemeinen Rechtsschutzprinzip (vgl. die grundsätzliche Regelung des Artikels 24 der Verfassung) ausnehmen kann. Als Folge hieraus hat das Verfassungsgericht die Maßnahmen des CSM der Überprüfbarkeit durch unabhängige Verwaltungsgerichte unterworfen (VerfassungsgerichtsE Nr. 14/ 1968 und 189/1992).

Eine weitere Bestimmung sah vor, dem CSM finanzielle Autonomie bei der Wahrnehmung seiner Aufgaben zuzuerkennen.

Die Rolle des Vizepräsidenten ist entscheidend für die Realisierung einer effektiven Autonomie des CSM. Es ist nicht von ungefähr, dass seine Rolle immer noch offen ist, noch ist es zufällig, dass die Reformprojekte, die eine Beschneidung der Zuständigkeiten und der Autonomie des CSM zum Ziel hatten, den Vizepräsidenten abschaffen wollten. Derzeit wird der Vizepräsident in geheimer Abstimmung während der ersten Sitzung des neu zusammengesetzten CSM gewählt. Jede Wahl erinnert an einen ungelösten Streit, der an sich nicht besonders bemerkenswert wäre, wenn er nicht den Kern des Verständnisses unterschiedlicher Konzeptionen des CSM und seiner offiziellen Vertreter berühren würde. Er dreht sich um die Legitimität einer - der Wahl vorgehenden - Debatte im Gremium, in der eine Begründung für die Wahl des Vizepräsidenten formuliert werden soll. Jene, die behaupten, eine solche Debatte sei legitim, betonen die politische Natur (im weitesten Sinne) der Wahl und ihre Bedeutung innerhalb des organischen Lebens des CSM; andere, die demgegenüber argumentieren, die Wahl müsse die erste Handlung des jeweils neu zusammentretenden CSM sein, geben zu be- 
denken, eine öffentliche Debatte würde das Wesen der gesetzlich vorgeschriebenen geheimen Wahl verletzen (zwar nicht formal, aber materiell) und den Vizepräsidenten lediglich zu einem Vertreter einer klar formulierten politischen Überzeugung machen. Die schließlich gefundene Kompromisslösung sieht die geheime Wahl ohne Debatte in der ersten offiziellen Sitzung vor, während in einer vorausgehenden nichtoffiziellen Sitzung in eher informeller Art über die Kandidaten diskutiert wird.

\section{Die Rolle des Justizministers}

Die Beziehungen zum Justizminister stellen einen weiteren Konfliktbereich im institutionellen Gefüge dar. Wie oben erwähnt ist der Justizminister laut Art. 110 der Verfassung verantwortlich für die Organisation der Justiz sowie die nachgeordneten Dienste. Die Abgrenzungen der Zuständigkeiten des CSM von jenen des Justizministers führen zu einem ständigen Kampf, in dessen Verlauf auch das Verfassungsgericht angerufen wurde.

Die Hauptstreitpunkte betrafen die Rolle der beiden Organe bei der Ernennung der Gerichtspräsidenten und der Leiter der Staatsanwaltschaften sowie die Ausweitung ministerieller Macht beim Erlass von Verordnungen zur Durchführung von Entscheidungen des CSM.

Die Entscheidung des Verfassungsgerichts (Erlass Nr. 379/1992) schreibt vor, dass bei Entscheidungen über Besetzung von Leitungsfunktionen der Minister in einer Weise hinzugezogen werden muss, die über eine bloß formale Anhörungspflicht hinausgeht und dem Grundsatz des Bemühens um loyales Zusammenwirken der betroffenen Institutionen entspricht. Die Letztentscheidung liegt jedoch in der Zuständigkeit des CSM.

Die Zuständigkeit des Ministers für Anwendungserlasse zu CSM-Entscheidungen beinhaltet nicht die Berechtigung, den Inhalt der Entscheidungen zu bewerten; es handelt sich lediglich um eine Pflicht, die nach Abschluss des Bemühens um gegenseitige Verständigung auszuüben ist, sofern keine wesentlichen Mängel der CSM-Entscheidung bestehen.

\section{Die Rolle des Obersten Justizrats (CSM) bei der Organisation der Behörden: das »Tabellen«-System}

In der Verantwortlichkeit für die Justiz kommt dem »Tabellen«-System eine maßgebliche Bedeutung zu. Bei dem »Tabellen «-System handelt es sich um ein Instrumentarium, das vom CSM über viele Jahre entwickelt worden ist, um das Fehlen normierter Regeln zur Geschäftsabwicklung (Zuweisung und Verteilung von Zuständigkeiten und Verfahren etc.) auszugleichen. Der Präsident eines Gerichts bzw. der Leiter einer Staatsanwaltschaft ist daher dazu aufgefordert, in regelmäßigen Abständen einen Organisationsplan zu entwerfen. Von diesem Plan hängt die Verteilung der Zuständigkeiten unter den Richtern und Staatsanwälten, also die Zuweisung von Rechtsbereichen und Verfahren (Geschäftsverteilungsplan), ab. Als »Tabelle« wird somit ein strukturiertes Programm bezeichnet, das Einteilungen und Aufstellungen beinhaltet. 
Es hat seine gegenwärtige Ausgestaltung in einem langen Prozess der Selbstverwaltung - ohne normative Vorgaben - erfahren. Das »Tabellen«-Programm wurde über einen großen Zeitraum entwickelt, um die Organisation aller mit der Rechtsprechung verknüpften Aufgaben nach einheitlichen Vorgaben zu gestalten, und zwar mit dem zweifachen Ziel, die Ermessensfreiheit der Gerichtspräsidenten und Leiter der Staatsanwaltschaften durch die Vorgabe von allgemein gültigen Richtlinien einzuschränken sowie die Organisationsabläufe durch die Anwendung einheitlicher Kriterien zu überwachen.

Zusammengefasst lässt sich sagen, dass dieses »Tabellen«-System die Aporie einer Justizorganisation manifestiert, die eine übersichtliche Gestaltung der Verwaltung sicher stellen soll, während sie gleichzeitig die Präsidenten und Leiter der Staatsanwaltschaften von politischer Verantwortung freistellen will, zumindest soweit sie verwaltend tätig werden. Es stellt das Bemühen dar, eine Reihe nicht ohne weiteres miteinander vereinbarer Interessen auszugleichen. Vor diesem Hintergrund wird deutlich, weshalb die Reform von 2005/2006 mit ihrem Bestreben, die Organisationsmacht (und die Entscheidung über die Strafverfolgung) in den Händen des Leiters der Staatsanwaltschaft zu konzentrieren, darauf abzielte, das »Tabellen «-System für die Staatsanwaltschaft abzuschaffen und nur noch für die Gerichte beizubehalten.

\section{Verschiedene Aspekte der Unabhängigkeit: Außergerichtliche Nebentätigkeiten}

Es muss betont werden, dass für den Bereich der justizexternen Tätigkeiten (zum Zwecke der Beratung öffentlicher Institutionen, Erfüllung von Lehraufträgen, für gutachterliche Tätigkeit, Berufungen zu supranationalen Behörden z. B. der EU) ebenfalls lange Zeit normierte Regelungen fehlten. Daher fühlte sich der CSM verpflichtet, diese Gesetzeslücke durch eigene Regelungen zu füllen. Der CSM sah sich dabei veranlasst, die beiden hierbei tangierten Interessenbereiche mit zunehmender Stringenz gegeneinander abzuwägen: Zum einen den Wunsch der betroffenen Richter und Staatsanwälte, ihren beruflichen Erfahrungsbereich nicht zuletzt auch aufgrund von staatsbürgerlicher Verantwortung erweitern zu können, zum anderen den Schutz ihrer Unabhängigkeit und Autonomie gegenüber jedem möglichen anderen Machtzentrum. Diese Einstellung des CSM ist keineswegs von allen Seiten gut geheißen worden, insbesondere deshalb, weil sie geeignet war, Möglichkeiten geheimer oder verborgener Einflussnahmen auszuschließen.

In gewisser Hinsicht scheint es paradox zu sein, dass schließlich ausgerechnet eine vom CSM verabschiedete Resolution den Ernennungen von Richtern bzw. Staatsanwälten zu besonders lukrativen Posten ein Ende setzte, nämlich wenn zu vermuten war, die Ausübung der fraglichen Tätigkeit könne zu einer Beeinträchtigung des Ansehens des Richters bzw. Staatsanwalts führen (z. B. bei Tätigkeiten als Schlichter oder Gutachter in Fällen von Vertragsauslegungen oder -gestaltungen, bei denen der Staat beteiligt ist), während Verwaltungsgerichte und das Parlament alles unternahmen, um die Folgen dieser wegweisenden Entwicklung zu unterbinden. 


\section{Das Verständnis von autonomer Verwaltung als ein von unten beginnender Kreis- lauf}

Autonome Verwaltung findet vornehmlich in der Verantwortung des CSM statt, aber sie gewinnt ihre Gestalt erst durch einen komplexen Kreislauf, dessen Ausgangspunkt der individuelle Richter bzw. Staatsanwalt ist. Dies ist keineswegs eine Rhetorik verbrämt demagogischer Natur. Richter und Staatsanwälte sind integraler Bestandteil eines komplexen Mechanismus, dessen Funktion darin besteht, Handlungen und Maßnahmen des CSM zu veranlassen. Diese Sichtweise, den einzelnen Richter bzw. Staatsanwalt als Ausgangspunkt des genannten Kreislaufes zu betrachten, ist unauflösbar verknüpft mit zwei Verfassungsprinzipien, eines ist ausdrücklich formuliert, während das andere aus der Summe der Verfassungsregeln abgeleitet werden muss: Richter bzw. Staatsanwälte werden nur aufgrund ihrer Funktionen voneinander unterschieden (Art. 107), und sie sind Ausdruck einer geteilten, die Justiz durchdringenden Macht (diffused power), also nicht abgeleitet von höheren Organisationsebenen innerhalb der Justiz. Diese Interaktion zwischen den Prinzipien hat weitreichende Folgen verschiedenster Art gehabt. Vorliegend sollte der Fokus auf die Gewährleistung der Unabhängigkeit des einzelnen Richters bzw. Staatsanwalts gerichtet sein, nicht nur gegenüber externem Druck, sondern auch gegenüber Einflüssen, die durch das Tätigsein innerhalb einer Organisationsstruktur bedingt sein können. Die Rolle des Richters bzw. Staatsanwalts ist nicht darauf beschränkt, seine Stimme im Rahmen von Verfahren zu erheben, deren politische und institutionelle Bedeutung bereits hinreichend erläutert wurde. Er ist vielmehr auch beauftragt, eine aktive Rolle bei der Vorbereitung wesentlicher Maßnahmen der Geschäftsorganisation zu übernehmen. Der Richter bzw. Staatsanwalt übt seine Rolle als Gründungsmitglied des Kreislaufs innerhalb einer autonomen Verwaltung aus, indem er auf die Gestaltung sowohl beabsichtigter Organisationsmaßnahmen als auch der Geschäftsverteilung Einfluss nimmt.

Auch den Präsidenten der Gerichte und Leitern der Staatsanwaltschaften kommt eine fundamentale Rolle zu. Das Verfassungsgericht hat vor kurzem die Bedeutung der Geschäftsführung in Anbetracht des unabhängigen Status der Justiz hervorgehoben (wobei auch die Zuständigkeit für die Aufstellung des Geschäftsverteilungsplans angesprochen wurde; VerfassungsgerichtsE Nr. 379/1992).

Die Rolle der Verwaltung beschränkt sich nicht nur auf das zugegebenermaßen entscheidende Erstellen des »Tabellen«-Systems (von dessen Planung über die BeschlieBung bis zur Anwendung); sie beinhaltet auch eine ständige Kommunikation mit den anderen Teilnehmern am Kreislauf der Geschäftsabwicklung, von den (auf Bezirksebene bestehenden) Justizräten ${ }^{1}$ bis zum CSM.

Die vom Exekutivbereich der Gerichte und Staatsanwaltschaften entworfenen Geschäftsverteilungspläne werden, nach deren Billigung in dem oben beschriebenen Verfahren, dem zuständigen Justizrat übermittelt, und dieser leitet ihn dann mit einer begründeten Stellungnahme an den CSM weiter. Die Mitglieder des Justizrats sind

1 Consigli Giudiziari, ein jeweils bei den Appellationsgerichten angesiedeltes Gremium, bestehend aus von Richtern gewählten Richtern. 
verpflichtet, in regelmäßigen Abständen Berichte über die in ihrem Zuständigkeitsbereich tätigen Richter und Staatsanwälte zu erstellen, die dann als Grundlage für die Beurteilung der richterlichen/staatsanwaltlichen Tätigkeit oder für eventuelle Beförderungen dienen. Beurteilungen werden zu unterschiedlichen Anlässen, von denen einer der wichtigsten die Übernahme von justizexternen Nebentätigkeiten (wie oben beschrieben) darstellt. Die Entscheidung obliegt dem CSM, aber die Präsidenten und Leiter der Staatsanwaltschaften haben ihre Meinung zur Kompatibilität der fraglichen Ernennung mit der originären Tätigkeit als Richter bzw. Staatsanwalts und - noch wichtiger - mit dessen Unabhängigkeit zu äußern.

Der Justizrat ist ein Organ, dessen Bedeutung zuweilen unterschätzt worden ist. Er ist nicht durch die Verfassung vorgeschrieben, sondern wurde als Ergebnis eines die Tätigkeiten des CSM regelnden Gesetzes geschaffen, und ist heute das eigentliche Verbindungsglied zwischen den Gerichten/Staatsanwaltschaften und dem CSM. Die Mitglieder des Justizrats werden von den Richtern und Staatsanwälten des jeweiligen Bezirks des Appellationsgerichts gewählt; er wird ergänzt durch Vertreter anderer juristischer Berufe. Jene, die immer wieder auf die Bedeutung der heterogenen Zusammensetzung des CSM hingewiesen haben, versuchten, die Justizräte auch für andere gesellschaftliche Beteiligungen zu öffnen, zunächst für Vertreter der Rechtsanwaltskammer (Ordine degli Avvocati, die in Italien eine öffentlich-rechtliche Körperschaft ist). Dieses Vorhaben ist auf Widerstand gestoßen: Mitunter lediglich auf Widerstand korporatistischer Art, ein anderes Mal aufgrund der Befürchtung, dass Partikularinteressen die Funktion der Justizräte beeinflussen könnten. Erst mit der Reform von 2005/ 2006 ist mit der Zulassung von Vertretern der Rechtsanwaltskammer wenigstens zu bestimmten Tätigkeitsbereichen der Justizräte ein erster Durchbruch gelungen.

Nach meiner Auffassung ist ein weiterer Schritt wünschenswert: Die Zulassung renommierter Juristen aus dem regionalen Umfeld, auf regionaler Basis gewählt, um so die Zusammensetzung des Justizrats gerade hinsichtlich seiner Organisationsverantwortung und bei seinem Bemühen um den Schutz der individuellen Unabhängigkeit zu ergänzen.

Vor kurzem (2008) ist mit der Schaffung eines Verwaltungsrates am Kassationsgericht ein weiterer bedeutender Fortschritt erzielt worden. Der Name mag sich geändert haben, aber nicht die Rolle und Zusammensetzung: Dieser Verwaltungsrat ist für das Kassationsgericht, was die Justizräte für den jeweiligen Bezirk der Appellationsgerichte sind. Es ist noch zu früh, die Folgen einer solchen Reform für den Obersten Gerichtshof zu bewerten. Jedenfalls ist mit diesem Verwaltungsrat am Kassationsgericht der Kreis der autonomen Verwaltung vervollständigt worden.

Zusammenfassend lässt sich feststellen, dass der CSM im institutionellen Zusammenspiel der Kräfte Italiens einen wesentlichen Schutz für die Autonomie und die Unabhängigkeit der Justiz geboten hat. Eine derartige Rolle ist nur dadurch möglich geworden, dass der CSM zur autorisierten Stimme und Vertretung der Justiz wurde, nicht zuletzt deshalb, weil er den legitimen Streit zwischen den Gewalten innerhalb institutionalisierter Grenzen zuließ, selbst in einem Klima, das von großen politischen Konflikten gekennzeichnet war. 
Ferner hat der CSM bei mehr als einer Gelegenheit ein Instrumentarium entwickelt, um mit unterschiedlichen, die Effizienz der Justiz betreffenden Problemen umzugehen, da er gezielter handeln konnte als ein in seinen Handlungsmöglichkeiten gespaltenes und oft unbewegliches Parlament.

Die wichtigste Entscheidung des CSM war es jedoch, jede Ebene der Justiz am Entscheidungsprozess zu beteiligen, um so eine autonome Verwaltung zu schaffen, die von unten, d.h. bei jedem einzelnen Richter beginnt. Dieses Handlungsmuster war darauf angelegt, die Rolle des einzelnen Richters als Gestaltungsmoment einer Vorstellung von Justiz als geteilte Macht zu sehen.

In diesem Zusammenhang ermöglichte das oben beschriebene »Tabellen«-System dem CSM und dem Justizminister (gemäß ihrer jeweiligen Zuständigkeit), Gerichte und Staatsanwaltschaften zu organisieren, und zwar mit einer Ordnungsvorgabe, die von der Orientierung geleitet war, eine Balance zwischen dem Interesse an Effizienz der Rechtsprechung und dem Interesse an der Bewahrung der Unabhängigkeit der Justiz zu erreichen.

Entscheidend ist, dass die von Erfahrung geprägte Gestaltung des CSM und seiner Aufgaben sowie die vielschichtige Natur des Rates (gewählt, aber nicht politisch verantwortlich; zusammengesetzt aus Laien und Richtern bzw. Staatsanwälten; mit dem Präsidenten der Republik als Vorsitzendem, aber einem gewählten Vizepräsidenten; ausgestattet mit der Kompetenz der Letztentscheidung hinsichtlich aller justizinternen Angelegenheiten, jedoch nicht ermächtigt, die eigenen Entscheidungen zwangsweise durchzusetzen) ihre Wirkungsmacht und Legitimität während einer Serie von Zusammenstößen zwischen Politik und Justiz bewiesen hat. Diese Wertung wird noch signifikanter, wenn man die immer wiederkehrende Berufung auf den »Vorrang des Gesetzes « berücksichtigt, als eine Art von Mantra, oft in einer sehr oberflächlichen Weise verstanden, gänzlich unberührt von der jahrhundertewährenden Debatte und Formulierungsbemühungen um die Herrschaft des Gesetzes.

In den hier geschilderten Kämpfen artikulieren sich grundlegende Unterschiede in der Auffassung von liberalen Institutionen und darin, wie die Balance zwischen den Staatsgewalten zu gestalten ist. Dies betrifft weit mehr als nur den CSM, nämlich die Idee der Justiz an sich und ihrer Rolle in einer komplexen, offenen Gesellschaft.

Italien präsentiert in dieser Hinsicht dank seiner Geschichte und seiner noch jungen Demokratie (mit schwachen liberalen Institutionen und Gewohnheiten) mehr als nur einzelne Wesensmerkmale. Auch wenn dies kein nachahmenswertes Modell ist, sollten doch die Erfahrungen meines Landes im Bemühen, sich vom organisierten Verbrechen und von Korruption zu befreien (dabei aber individuelle und kollektive Rechte zu schützen) Gegenstand aufmerksamer Beobachtung sein, die anderen Ländern Beispiele und Erfahrungen positiver wie negativer Natur bieten können. Für mich ist es von außerordentlicher Bedeutung, dass Länder mit unterschiedlichen institutionellen Systemen die Vorteile eines starken, autonomen Rates (CSM), als fundamentale Voraussetzung für die Stärkung einer unabhängigen Justiz diskutieren. 